\title{
Case Report: Scapholunate Instability Following the Surgical Excision of Dorsal Ganglion Cyst of the Wrist: A Case Report
}

\author{
Hooman Shariatzade $^{1}$ (D), Mohsen Barkam ${ }^{1 *}$ id, Alireza Saied ${ }^{2}$, Alireza Akbarzadeh Arab ${ }^{1}$ \\ 1. Bone and Joint Reconstruction Research Center, Shafa Orthopedic Hospital, Iran University of Medical Sciences, Tehran, Iran \\ 2. Dr. Bahonar Hospital, School of Medicine, Kerman University of Medical Sciences, Kerman, Iran.
}

\begin{tabular}{|l|l}
\hline $\begin{array}{l}\text { Use your device to scan } \\
\text { and read the article online }\end{array}$ & $\begin{array}{l}\text { Crtation Shariatzade H, Barkam M, Saied AR, Akbarzadeh Arab AR. Scapholunate Instability Following the Surgical Exci- } \\
\text { sion of Dorsal Ganglion Cyst of the Wrist: A Case Report. Journal of Research in Orthopedic Science. 2021; 8(2):95-100. http:// } \\
\text { dx.doi.org/10.32598/JROSJ.8.2.723.2 }\end{array}$ \\
dol: $:$ http://dx.doi.org/10.32598/JROSJ.8.2.723.2
\end{tabular}

\section{(c) (i) (5)}

Article info:

Received: 18 Jan 2021

Revised: 05 Feb 2021

Accepted: 03 Mar 2021

Available Online: 01 May 2021

Keywords:

Ganglion cyst, Wrist, Scapholunate instability, Joint Instability

\begin{abstract}
A B S T RA C T
Ganglion cysts of the dorsal wrist are generally attached to the scapholunate interosseous ligament, and surgical removal could injure this ligament. Such injury could rarely result in postoperative scapholunate instability. To date, a few cases of scapholunate instability following the excision of the dorsal ganglion cyst of the wrist have been reported. In this report, we present a 23-year-old man with scapholunate instability following the surgical resection of the dorsal ganglion cyst of his wrist. The instability was treated with open reduction and reconstruction. One year follow-up of the patient was event-free. The patient had no pain and limitation and resumed his preoperative activities. According to this case, the iatrogenic or pre-existing nature of scapholunate instability following the surgical excision of the dorsal ganglion cyst of the wrist cannot be determined. However, the patients should be informed of this complication before undergoing surgery.
\end{abstract}

\section{Introduction}

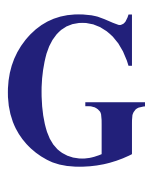

anglion cysts are benign synovial lesions commonly found within the wrist and hand. The majority of ganglion cysts are asymptomatic. However, they could be presented with pain, weakness, and tenderness. Non-surgical treatment of ganglion cysts has a high risk of recurrence. Surgical excision has a considerably smaller risk of recurrence, but it is associated with various postoperative complications with a rate ranging from $0 \%$ to $56 \%$, including infection, stiffness, neuroma, and keloid formation $[1,2]$.

Scapholunate instability is a rare complication following the surgical excision of the dorsal ganglion cyst of the wrist. Since ganglion cysts of this site originate from the scapholunate joint and are generally attached to the scapholunate interosseous ligament, surgical excision includes removing the attachments to the scapholunate ligament to minimize the risk of recurrence. The inva-

\section{"Corresponding Author:}

Mohsen Barkam, MD.

Address: Bone and Joint Reconstruction Research Center, Shafa Orthopedic Hospital, Iran University of Medical Sciences, Tehran, Iran

Phone: +98 (913) 3970840

E-mail: drbarkam63@gmail.com 
sion of the scapholunate ligament could cause scapholunate instability [3].

Knowing the potential complications following the surgical excision of the ganglion cyst is necessary for optimizing the result of this operation. In this study, we report a case of scapholunate instability in a 23-year-old male following the surgical resection of a ganglion cyst of a dorsal wrist.

\section{Case Presentation}

A 23-year-old right-handed male was referred to our center with a diagnosis of scapholunate dissociation 8 months after the surgical excision of the dorsal ganglion cyst of his right wrist. No splint was implemented after the surgery as it could cause prolonged joint stiffness and decreased range of motion [4]. The patient started active wrist movements after two weeks. However, three weeks after the surgery, the patient reported pain at the dorsal wrist when doing the wrist movements. The pain aggravated over the following months, and a clicking sound was also felt when moving the wrist. Six months after the surgery, the wrist Range of Movements (ROM) became limited and painful, particularly at dorsiflexion.

Initial clinical examination at our center revealed slight swelling and tenderness of the scapholunate area in the dorsal side. The transverse scar of the cyst excision was noted on the dorsal wrist (Figure 1).

According to the description of the initial surgery provided in the patient's file summary and telephone contact with the first surgeon, it was determined that the mass excision and capsulotomy were done as the index surgery to prevent the recurrence of the lesion. Intraoperatively, it turned out to be a ganglion cyst confirmed by the pathology report. The first surgeon mentioned an uncomplicated surgery without any particular iatrogenic error.

The wrist dorsiflexion and palmer flexion were $20^{\circ}$ and $65^{\circ}$, respectively. The wrist supination and pronation were nearly normal. The ballottement test of scapholunate was positive and associated with severe pain. Watson's test (scaphoid shift test) was also positive and painful. The grip strength of the involved hand was reduced compared with the contralateral hand. The patient reported no history of postoperative trauma or severe wrist-twisting. Anteroposterior and lateral radiographs of the wrist were consistent with the diagnosis of scapholunate dissociation. The scapholunate distance was approximately $7 \mathrm{~mm}$. Evidence of dorsal intercalated segment instability was present in the lateral view radio- graph with a scapholunate angle of $90^{\circ}$, while no sign of scapholunate joint injury was available in preoperative radiographs of the patient (Figure 2).

In the operation room, a 5-cm longitudinal incision was made over the dorsal wrist of the patients. The extensor retinaculum was opened using a Z-cut, and the approach continued through the third and fourth extensors. The remaining dorsal capsule of the wrist was preserved. Hypertrophic synovium and fibrous tissues were extracted. Scapholunate ligament rupture was evident. We first reduced the scapholunate joint with a $1.5-\mathrm{mm}$ pin. Then the ends of the torn scapholunate ligaments were trimmed. The remaining scapholunate ligament was not enough for the repair. Owing to scapholunate instability, reconstruction of the scapholunate ligament was indicated. For this purpose, a strip of flexor carpi radialis tendon was obtained through a 4-cm longitudinal excision on the volar aspect of the wrist while preserving the portion connected to the second metacarpal. Scapholunate reconstruction was done using the modified Brunelli procedure as earlier described [5] (Figure 3).

After the surgery, a short forearm cast was implemented for the patient. Radiographic evaluation revealed the normal alignment of the scapholunate (Figure 4). Two weeks after the surgery, the sutures were removed. The cast was

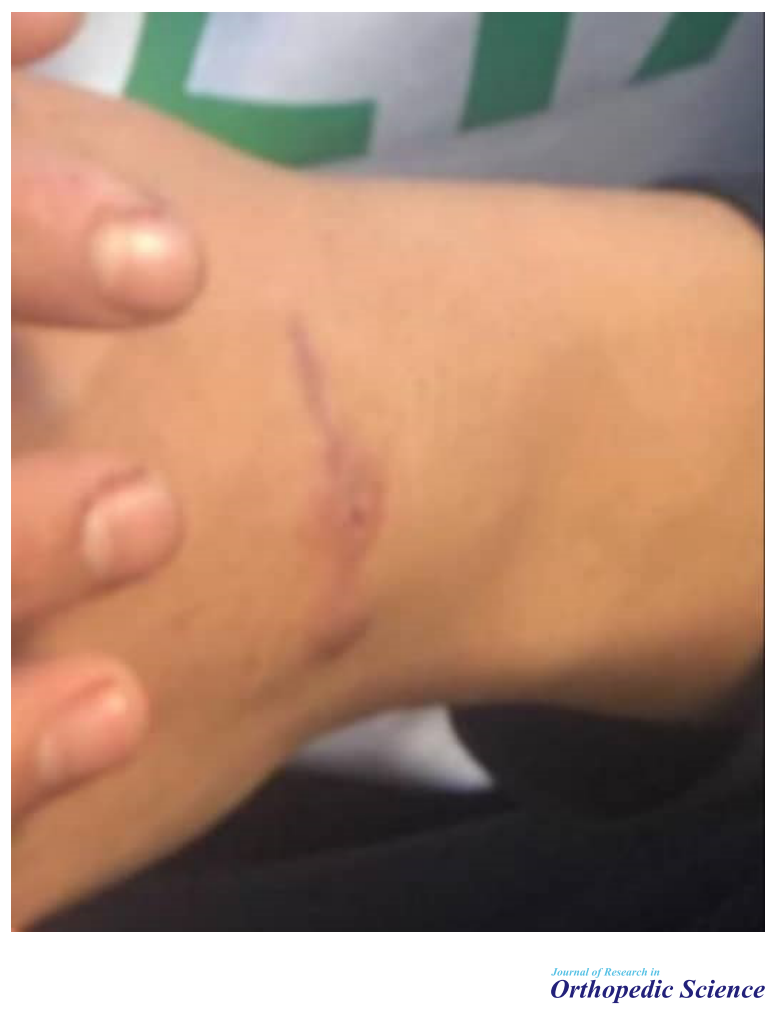

Figure 1. The transverse scar of the cyst excision from the primary surgery 

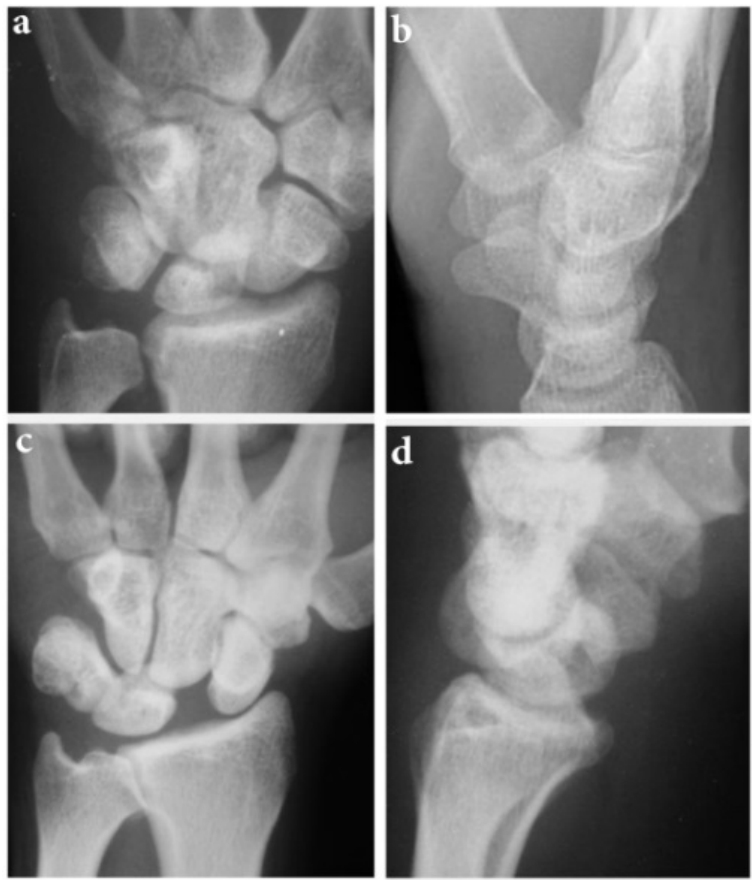

Figure 2. Plain radiographies of wrist

Orthopedic Science

(a) Anteroposterior and (b) Lateral radiograph of the wrist before the excision of the cyst with no sign of scapholunate Joint injury; (c) Anteroposterior and (d) Lateral radiograph of the wrist eight months after the excision of the cyst showing the dorsal intercalated segment instability.

removed six weeks after the surgery. Six weeks after the surgery, the pins were removed (Figure 5) at an outpatient facility, and then active wrist ROM was started. Physical therapy was started three months after the surgery.

Figure 6 reveals the postoperative photograph of the patient's wrist showing the transverse scare of the first

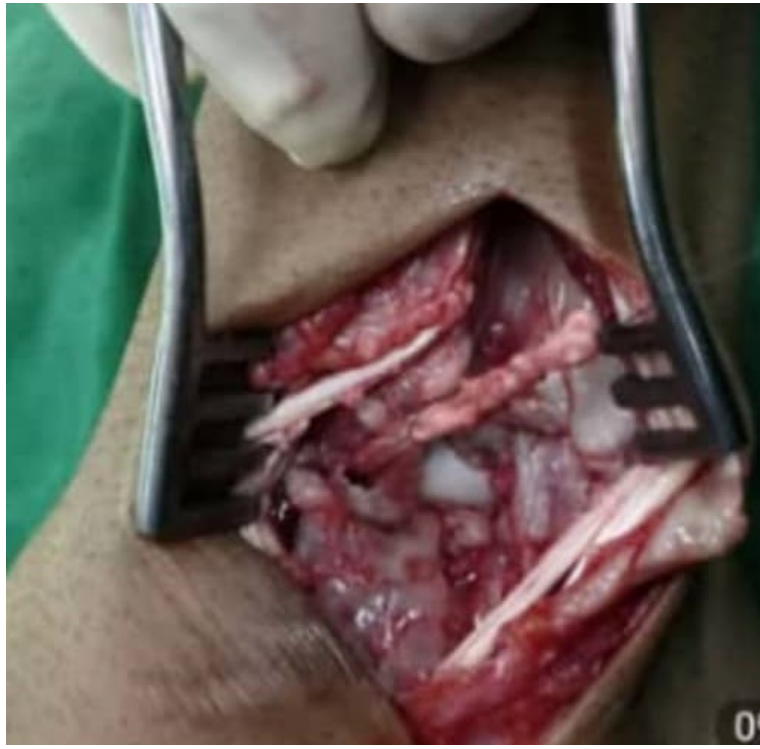

Orthopedic Science

Figure 3. Intraoperative photograph

Scapholunate reconstruction using the brunelli procedure.

surgery (cyst excision) crossed by the longitudinal scare of the second surgery (scapholunate ligament reconstruction). One year after the surgery, the patient was pain-free. The ballottement test was negative. The scaphoid was stable in clinical examination. The grip strength reached $80 \%$ of the contralateral hand. The final dorsiflexion and palmar flection were $60^{\circ}$ and $68^{\circ}$, respectively.

\section{Discussion}

Surgical removal of ganglion cysts of the wrist could result in serious postoperative complications, particularly if they were at the vicinity of the crucial structures such as the scapholunate ligament. In this study, we re-
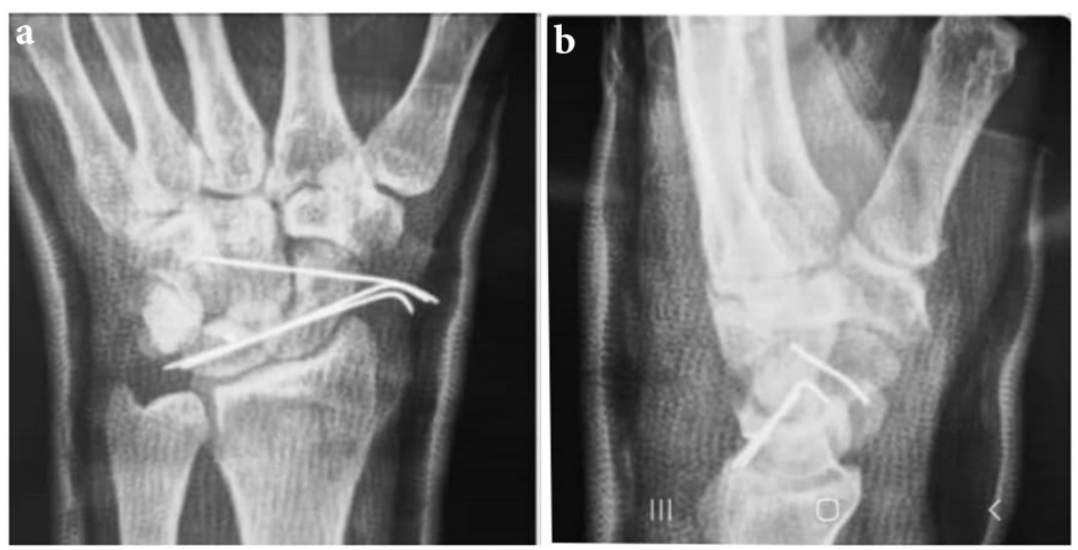

Orthopedic Science

Figure 4. Anteroposterior (a) and Lateral (b) radiograph of the wrist immediately after ligament reconstruction surgery

The alignment of the scapholunate is normal. 


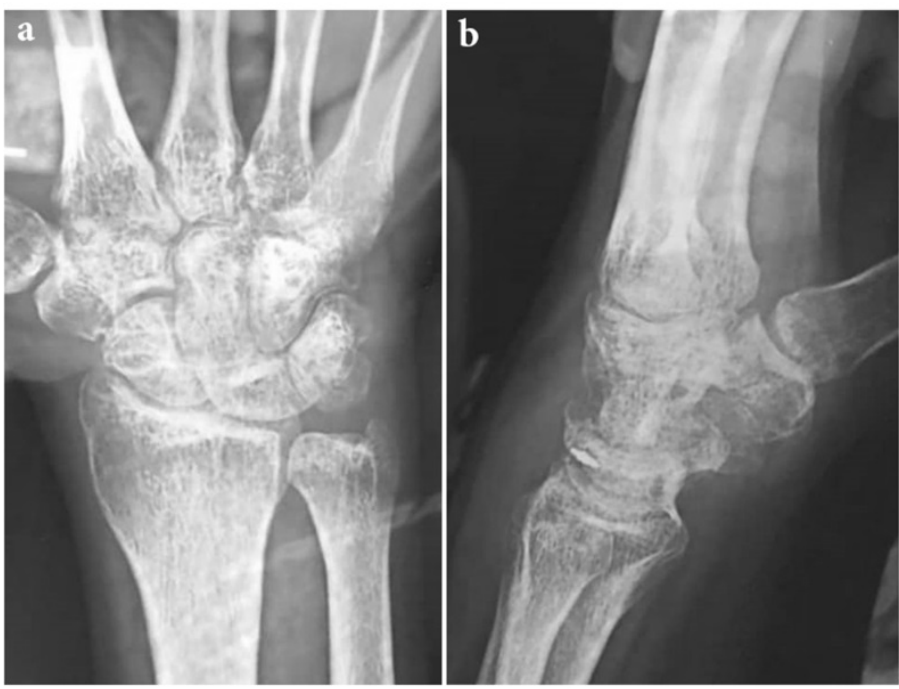

Figure 5. Anteroposterior (a) and Lateral (b) radiograph of the wrist joint immediately after pin removal Orthopedic Science

ported a case of scapholunate instability in a 23-year-old male following the surgical removal of the dorsal ganglion cysts of the wrist. This complication has been rarely reported in earlier studies $[3,6,7]$.

Duncan and Levis reported a case of scapholunate instability in a 38-year-old male following the surgical excision of the dorsal ganglion cyst of the wrist. After the excision, the wrist was immobilized with a splint for three weeks, and progressive wrist ROM was started afterward. The patient presented four months after the surgery following a pop in the wrist. Clinical examination revealed a dorsal wrist tenderness that was associated with a scaphoid subluxation in the roentographs. Since the ligament was disrupted, it was reconstructed instead of repair. Nine months after the operation, the patient resumed his routine activities with no limitation [7]. We did not immobilize the wrist after the excision of the cyst. Similarly, ligament reconstruction was successful in the treatment of scapholunate instability in our patient.

Mehdian and McKee presented a case of scapholunate instability following surgical excision of a dorsal wrist ganglion in a young patient who had a mild twisting injury. It was accompanied by limited and painful wrist ROM, a clicking sound, mild swelling, and tenderness on the scapholunate joint. The patient was treated with open reduction and ligamentous repair that resulted in complete satisfaction of patients and returning to all previous activities. The preoperative radiographs of the wrist were not available to rule out any abnormality of the scapholunate joint before the excision of the cyst [3]. The case presented here had no minor trauma or twisting. Therefore, the incidence of scapholunate instability following the excision of a dorsal wrist ganglion is not necessarily trauma-associated. Complete pre-excision radiographic records were available for our case, showing no scapholunate abnormality before removing the cyst.

Hwang et al. clinically and radiographically reviewed 18 patients with dorsal carpal ganglion cyst associated with a positive scaphoid shift test. After removing the cyst, all wrists were immobilized in $20^{\circ}$ of dorsiflexion using a bulky compressive dressing. No signs and symptoms of scapholunate instability were detected afterward. They mentioned that holding the wrist at $20^{\circ}$ for two weeks could reduce the rate of scapholunate insta-

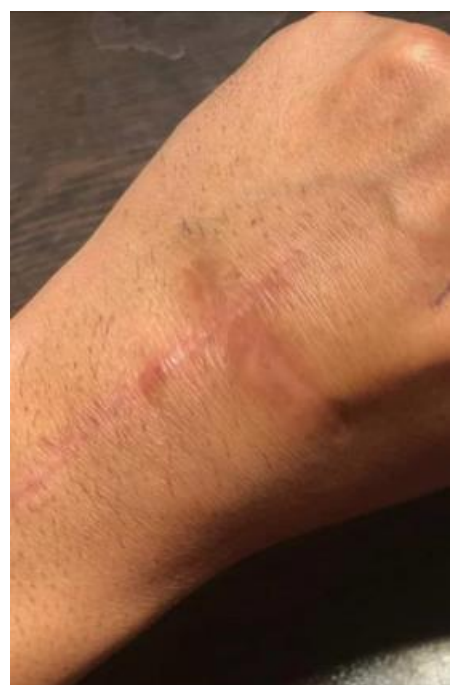

Orthopedic Science

Figure 6. A one-year postoperative figure

he transverse scare of the first surgery (cyst excision) crossed by the longitudinal scare of the second surgery (scapholunate ligament reconstruction). 
bility following the removal of the dorsal ganglion cyst of the wrist [6]. The wrist was not held in $20^{\circ}$ dorsiflexion in our case.

Rizzo et al. evaluated the outcomes of arthroscopic resection of dorsal wrist ganglions in 41 patients. The average follow-up period was 47.8 months. Recurrence of ganglion occurred in two patients (4.9\%). During the follow-up, no significant intraoperative or postoperative complications were recorded. Scapholunate instability was not noted in any patient. They concluded that arthroscopic ganglionectomy is a safe and effective alternative to open surgery that reduces the risk of scapholunate instability through a more controlled resection of the cyst while protecting the scapholunate interosseous ligament [8]. However, other studies have reported scapholunate instability following the arthroscopic ganglionectomy of the dorsal wrist $[9,10]$. Although early results of arthroscopic ganglionectomy of the dorsal wrist have been promising, the available inconsistencies require further studies comparing the rate of scapholunate instability following the arthroscopic and open removal of ganglion cysts of the dorsal wrist.

A preoperative positive scaphoid shift test was noticed in all patients of the Hwang study [6]. In addition, our patient had no history of minor trauma or twisting, as noted in other reports [3]. This information suggests that a dynamic scapholunate instability might be present before the surgery, turning into a static form after the surgery if the wrist movements are started early, before giving the ligament enough time to improve itself at $20^{\circ}$ dorsiflexion, as we did. In this case, static scapholunate instability could be detected in postoperative radiographs, while no evidence of instability could be seen in preoperative imaging. In other words, postoperative scapholunate instability cannot be considered a surgical complication alone. Whatever the reason, minimizing iatrogenic errors predisposing scapholunate instability such as cauterization, extensive deep dissection, etc. could diminish the rate of this complication after cyst removal. Immobilizing the wrist in a splint in $20^{\circ}$ dorsiflexion might also reduce the rate of scapholunate instability in this setting. However, these recommendations should be tested in further studies with larger sample size.

Altogether, surgical removal of ganglion cyst of the dorsal wrist could result in scapholunate instability owing to intraoperative injury of the scapholunate ligament. Therefore, surgeons should know this complication and minimize intraoperative injuries to this ligament, as well as monitor this instability following the surgery. To date, the iatrogenic or pre-existing nature of this complica- tion is not understood. Whatever the reason, the patients should be informed of this complication before undergoing surgery.

\section{Ethical Considerations}

\section{Compliance with ethical guidelines}

The patient was assured about the confidentiality of his information and medical data for publication.

\section{Funding}

This research did not receive any grant from funding agencies in the public, commercial, or non-profit sectors.

\section{Authors' contributions}

Supervision: Hooman Shariatzade; Writing - original draft: Mohsen Barkam; Data collection: Alireza Saied; Writing - review \& editing: Alireza Akbarzadeh Arab.

\section{Conflict of interest}

The authors declared no conflict of interest.

\section{References}

[1] Suen M, Fung B, Lung CP. Treatment of ganglion cysts. ISRN Orthop. 2013; 2013:940615. [DOI:10.1155/2013/940615]

[2] Dehghani H, Khadem M, Bahari M. Elbow region ganglion cyst: A case report and brief review of literature. J Res Orthop Sci. 2020; 7(2):87-92. [DOI:10.32598/JROSJ.7.2.619.1]

[3] Mehdian H, McKee MD. Scapholunate instability following dorsal wrist ganglion excision: A case report. Iowa Orthop J. 2005; 25:203-6. [PMID] [PMCID]

[4] Gude W, Morelli V. Ganglion cysts of the wrist: Pathophysiology, clinical picture, and management. Curr Rev Musculoskelet Med. 2008; 1(3-4):205-11. [DOI:10.1007/s12178-008-9033-4]

[5] Garcia-Elias M, Lluch AL, Stanley JK. Three-ligament tenodesis for the treatment of scapholunate dissociation: Indications and surgical technique. J Hand Surg Am. 2006; 31(1):125-34. [DOI:10.1016/j.jhsa.2005.10.011]

[6] Hwang JJ, Goldfarb CA, Gelberman RH, Boyer MI. The effect of dorsal carpal ganglion excision on the scaphoid shift test. J Hand Surg Br. 1999; 24(1):106-8. [DOI:10.1016/S02667681(99)90053-0]

[7] Duncan KH, Lewis Jr RC. Scapholunate instability following ganglion cyst excision. A case report. Clin Orthop Relat Res. 1988; (228):250-3. [DOI:10.1097/00003086-198803000-00038] 
[8] Rizzo M, Berger RA, Steinmann SP, Bishop AT. Arthroscopic resection in the management of dorsal wrist ganglions: Results with a minimum 2-year follow-up period. J Hand Surg Am. 2004; 29(1):59-62. [DOI:10.1016/j.jhsa.2003.10.018]

[9] Slutsky DJ, Nagle DJ. Wrist arthroscopy: Current concepts. J Hand Surg Am. 2008; 33(7):1228-44. [DOI:10.1016/j. jhsa.2008.07.015]

[10] Borisch N. Arthroscopic resection of occult dorsal wrist ganglia. Arch Orthop Trauma Surg. 2016; 136:1473-80. [DOI:10.1007/s00402-016-2539-0] 